УДК 531

\title{
Study of the Non-isothermal Coupled Problem with Mixed Boundary Conditions in a Thin Domain with Friction Law
}

\author{
Abdelkader Saadallah* \\ Hamid Benseridi ${ }^{\dagger}$ \\ Mourad Dilmi ${ }^{\ddagger}$ \\ Applied Mathematics Laboratory, Department of Mathematics \\ Setif I-University, 19000 \\ Algeria
}

Received 06.04.2018, received in revised form 06.07.2018, accepted 06.08.2018

This paper deals with the asymptotic behavior of a coupled system involving of an incompressible Bingham fluid and the equation of the heat energy, in a three-dimensional bounded domain with Tresca free boundary friction conditions. First we prove the existence and uniqueness results for the weak solution. Second, we show the strong convergence of the velocity and the temperature. Then a specific Reynolds limit equation is obtained, and the uniqueness of the limit velocity and temperature are proved.

Keywords: asymptotic approach, boundary conditions, Coupled problem, Fourier law, non-isothermal Bingham fluid, Tresca law, Reynolds equation.

DOI: 10.17516/1997-1397-2018-11-6-738-752.

\section{Introduction}

In many problems, which study the asymptotic behavior for a problem of continuum mechanics in a thin domain, we transform the problem into an equivalent problem on a domain $\Omega$ independent of the parameter $\varepsilon$. Specifically, the case of Bingham fluid has been studied by many authors, for example : the analysis of the Bingham fluid flow variational inequality was carried out in [9], where the authors investigated the existence, uniqueness and regularity of the solution for the steady and in-stationary flows in a reservoir. Existence and extra regularity results for the $d$-dimensional Bingham fluid flow problem with Dirichlet boundary conditions are also studied in $[11,12]$. The numerical solution of the stationary Bingham fluid flow problem is studied in $[6,7,13]$. The study of the a nonlinear boundary value problem governed by partial differential equations which describe the evolution of nonlinear elastic materials has been considered in [1]. In [10], the author has given in the last chapter of his doctoral thesis the asymptotic behavior of a Bingham fluid in a thin domain. Unfortunately this work is not done due to the difficulty encountered in this study which resides on the choice of test functions because of the boundary conditions imposed. Then in [5], the authors studied the same problem, in which, only the Dirichlet conditions on the boundary have been considered. The authors in [8] have proved the asymptotic analysis of a isothermal Bingham fluid in a thin domain with non linear Tresca boundary conditions.

In this present paper, we further the research of [8] on the asymptotic behavior of a Bingham fluid in a thin domain $\Omega^{\varepsilon} \subset \mathbb{R}^{3}$ with boundary $\Gamma^{\varepsilon}=\bar{\Gamma}_{1}^{\varepsilon} \cup \bar{\Gamma}_{L}^{\varepsilon} \cup \bar{\omega}$. However, this time we consider a coupled problem which describes the motion of an incompressible fluid in a thin domain,

\footnotetext{
*saadmath2009@gmail.com

$\dagger$ benseridi@yahoo.fr

${ }^{\ddagger}$ mouraddil@yahoo.fr

(c) Siberian Federal University. All rights reserved
} 
governed by a coupled system of the equation of motion and the equation of the heat energy, obtained by using Fourier's law and neglecting the dissipation term. We consider Dirichlet boundary conditions on $\bar{\Gamma}_{1}^{\varepsilon} \cup \bar{\Gamma}_{L}^{\varepsilon}$, where $\bar{\Gamma}_{L}^{\varepsilon}$ is the lateral one, the Fourier boundary condition at the top surface $\bar{\Gamma}_{1}^{\varepsilon}$, finally, a nonlinear Tresca interface condition and homogeneous Neumann condition for the temperature at the bottom one $\omega$. The weak form of the problem is a variational equality. The small change of variable $z=\frac{x_{3}}{\varepsilon}$, transforms the initial problem posed in the domain $\Omega^{\varepsilon}$ into a new problem posed on a fixed domain $\Omega$ independent of the parameter $\varepsilon$. We prove some estimates on the displacement and temperature independent of the small parameter. The passage to the limit on $\varepsilon$, permits us to obtain a weak form of the Reynolds equation, give a lower-dimensional Bingham law, prevalent in engineering literature and the uniqueness of the solution $\left(u^{*}, T^{*}\right)$.

This article is organized as follows. In Section 1, we recall the weak formulation of our coupled problem considered. Some estimates and convergence theorem by using the Korn and Poincaré inequalities (developed recently in Refs $[3,4]$ ) are given in Section 2. The limit problem with a specific weak form of the Reynolds equation, the uniqueness of the limit velocity and temperature are given in Section 3.

\section{Problem statement and variational formulation}

Let $\omega$ be fixed region in the plane $x^{\prime}=\left(x_{1}, x_{2}\right) \in \mathbb{R}^{2}$. We suppose that $\omega$ has a Lipschitz boundary and is the bottom of the fluid domain. The upper surface $\Gamma_{1}^{\varepsilon}$ is defined by $x_{3}=\varepsilon h\left(x^{\prime}\right)$ where $(0<\varepsilon<1)$ is a small parameter that will tend to zero and $h$ a smooth bounded function such that $0<\underline{h} \leqslant h\left(x^{\prime}\right) \leqslant \bar{h}$ for all $\left(x^{\prime}, 0\right)$ in $\omega$. We denote by $\Omega^{\varepsilon}$ the domain of the flow:

$$
\Omega^{\varepsilon}=\left\{x=\left(x^{\prime}, x_{3}\right) \in \mathbb{R}^{3}:\left(x^{\prime}, 0\right) \in \omega, 0<x_{3}<\varepsilon h\left(x^{\prime}\right)\right\} .
$$

The boundary of $\Omega^{\varepsilon}$ is $\Gamma^{\varepsilon}$. We have $\Gamma^{\varepsilon}=\bar{\Gamma}_{1}^{\varepsilon} \cup \bar{\Gamma}_{L}^{\varepsilon} \cup \bar{\omega}$ where $\Gamma_{L}^{\varepsilon}$ is the lateral boundary.

Let $\sigma^{\varepsilon}$ denotes the total Cauchy stress tensor: $\sigma^{\varepsilon}=-p^{\varepsilon} I+\sigma^{D, \varepsilon}$, where $\sigma^{D, \varepsilon}$ denotes its deviatoric part, and $p^{\varepsilon}$ the pressure. The fluid is supposed to be viscoplastic, and the relation between $\sigma^{D, \varepsilon}$ and $D\left(u^{\varepsilon}\right)$ is given by the Bingham model:

$$
\begin{cases}\sigma^{D, \varepsilon}=\alpha^{\varepsilon} \frac{D\left(u^{\varepsilon}\right)}{\left|D\left(u^{\varepsilon}\right)\right|}+2 \mu D\left(u^{\varepsilon}\right), & \text { when } D\left(u^{\varepsilon}\right) \neq 0 ; \\ \left|\sigma^{D, \varepsilon}\right| \leqslant \alpha^{\varepsilon}, & \text { when } D\left(u^{\varepsilon}\right)=0,\end{cases}
$$

or equivalently:

$$
D\left(u^{\varepsilon}\right)=\left\{\begin{array}{cl}
\frac{1}{2 \mu}\left(1-\frac{\alpha^{\varepsilon}}{\left|\sigma^{D, \varepsilon}\right|}\right) \sigma^{D, \varepsilon} & \text { when }\left|\sigma^{D, \varepsilon}\right|>\alpha^{\varepsilon}, \\
0 & \text { otherwise. }
\end{array}\right.
$$

here $\alpha^{\varepsilon} \geqslant 0$ is the yield stress, $\mu>0$ is the constant viscosity, $u^{\varepsilon}$ is the velocity field and $D\left(u^{\varepsilon}\right)=\frac{1}{2}\left(\nabla u^{\varepsilon}+\left(\nabla u^{\varepsilon}\right)^{T}\right)$. For any tensor $\tau=\left(\tau_{i j}\right)$, the notation $|\tau|$ represents the matrix norm: $|\tau|=\frac{1}{\sqrt{2}}\left(\sum_{i, j} \tau_{i j}\right)^{\frac{1}{2}}$.

- The law of conservation of momentum

$$
-\operatorname{div}\left(\sigma^{\varepsilon}\right)=f^{\varepsilon} \quad \text { in } \Omega^{\varepsilon} .
$$

where $f^{\varepsilon}=\left(f_{i}^{\varepsilon}\right)_{1 \leqslant i \leqslant 3}$, denotes the body forces. 
- The equation of the heat energy

$$
-\frac{\partial}{\partial x_{i}}\left(G^{\varepsilon} \frac{\partial T^{\varepsilon}}{\partial x_{i}}\right)=2 \mu^{\varepsilon}\left(T^{\varepsilon}\right) d_{i j}\left(u^{\varepsilon}\right) d_{i j}\left(u^{\varepsilon}\right)+\sqrt{2} \alpha^{\varepsilon}\left|D\left(u^{\varepsilon}\right)\right|+r^{\varepsilon}\left(T^{\varepsilon}\right) \text { in } \Omega^{\varepsilon}
$$

obtained by using Fourier's law in which we neglect the dissipation term, where $G^{\varepsilon}=G^{\varepsilon}(x)$ is the thermal conductivity and $r^{\varepsilon}\left(T^{\varepsilon}\right)$ is the heat sources (see [9]).

- The incompressibility equation

$$
\operatorname{div}\left(u^{\varepsilon}\right)=0 \quad \text { in } \Omega^{\varepsilon} .
$$

Our boundary conditions are describe as

- At the surface $\Gamma_{1}^{\varepsilon}$ we assume

$$
\left.\begin{array}{c}
\sigma_{\tau}\left(u^{\varepsilon}\right)+l^{\varepsilon} u^{\varepsilon}=0 \\
u^{\varepsilon} \cdot n=0
\end{array}\right\} \text { on } \Gamma_{1}^{\varepsilon}
$$

where $l^{\varepsilon}>0$ on which we will bring precisions.

- On $\Gamma_{L}^{\varepsilon}$, the velocity is known and is parallel to the $\omega$-plane

$$
u^{\varepsilon}=0, \text { on } \Gamma_{L}^{\varepsilon}
$$

- On $\omega$, there is no-flux condition across $\omega$ so that

$$
u^{\varepsilon} \cdot n=0
$$

the tangential velocity on $\omega$ is unknown and satisfies Tresca boundary conditions with friction coefficient $k^{\varepsilon}$ (as [9]):

$$
\left.\begin{array}{l}
\left|\sigma_{\tau}^{\varepsilon}\right|<k^{\varepsilon} \Rightarrow u_{\tau}^{\varepsilon}=0 \\
\left|\sigma_{\tau}^{\varepsilon}\right|=k^{\varepsilon} \Rightarrow \exists \lambda \geqslant 0 u_{\tau}^{\varepsilon}=-\lambda \sigma_{\tau}^{\varepsilon}
\end{array}\right\} \text { on } \omega
$$

Here $n=\left(n_{1}, n_{2}, n_{3}\right)$ is the unit outward normal to $\Gamma^{\varepsilon}$, and

$$
u_{n}^{\varepsilon}=u^{\varepsilon} . n, \quad u_{\tau}^{\varepsilon}=u^{\varepsilon}-u_{n}^{\varepsilon} . n, \quad \sigma_{n}^{\varepsilon}=\left(\sigma^{\varepsilon} . n\right) . n \text { and } \sigma_{\tau}^{\varepsilon}=\sigma^{\varepsilon} \cdot n-\left(\sigma_{n}^{\varepsilon}\right) . n
$$

are, respectively, the normal and the tangential velocity on $\omega$, and the components of the normal and the tangential stress tensor on $\omega$.

For the temperature, we suppose that

$$
\begin{aligned}
T^{\varepsilon} & =0 \text { on } \Gamma_{1}^{\varepsilon} \cup \Gamma_{L}^{\varepsilon}, \\
\frac{\partial T^{\varepsilon}}{\partial n} & =0 \text { on } \omega .
\end{aligned}
$$

To get a weak formulation, we introduce :

$$
\begin{aligned}
K^{\varepsilon} & =\left\{\phi \in H^{1}\left(\Omega^{\varepsilon}\right)^{3}: \phi=0 \text { on } \Gamma_{L}^{\varepsilon}, \phi . n=0 \text { on } \omega \cup \Gamma_{1}^{\varepsilon}\right\}, \\
K_{d i v}^{\varepsilon} & =\left\{\phi \in K^{\varepsilon}: \operatorname{div}(\phi)=0\right\}, \\
L_{0}^{2}\left(\Omega^{\varepsilon}\right) & =\left\{q \in L^{2}\left(\Omega^{\varepsilon}\right): \int_{\Omega^{\varepsilon}} q d x=0\right\} \\
H_{\Gamma_{1}^{\varepsilon} \cup \Gamma_{L}^{\varepsilon}}^{1}\left(\Omega^{\varepsilon}\right) & =\left\{\varphi \in H^{1}\left(\Omega^{\varepsilon}\right): \varphi=0 \text { on } \Gamma_{1}^{\varepsilon} \cup \Gamma_{L}^{\varepsilon}\right\} .
\end{aligned}
$$

A formal application of Green's formula, using (2.1)-(2.9) leads to the weak formulation: 
Find $u^{\varepsilon} \in K_{\text {div }}^{\varepsilon}, p^{\varepsilon} \in L_{0}^{2}\left(\Omega^{\varepsilon}\right)$ and $T^{\varepsilon} \in H_{\Gamma_{1} \cup \Gamma_{L}}^{1}\left(\Omega^{\varepsilon}\right)$ such that

$$
\begin{gathered}
a\left(T^{\varepsilon} ; u^{\varepsilon}, \varphi-u^{\varepsilon}\right)-\left(p^{\varepsilon}, \operatorname{div} \varphi\right)+l^{\varepsilon} \int_{\Gamma_{1}^{\varepsilon}} u^{\varepsilon}\left(\varphi-u^{\varepsilon}\right) d \tau+J(\varphi)-J\left(u^{\varepsilon}\right) \geqslant \\
\geqslant\left(f^{\varepsilon}, \varphi-u^{\varepsilon}\right), \quad \forall \varphi \in K^{\varepsilon}\left(\Omega^{\varepsilon}\right), \\
b\left(T^{\varepsilon}, \varphi\right)=c\left(u^{\varepsilon} ; T^{\varepsilon}, \varphi\right), \quad \forall \varphi \in H_{\Gamma_{1} \cup \Gamma_{L}}^{1}\left(\Omega^{\varepsilon}\right),
\end{gathered}
$$

where

$$
\begin{aligned}
a\left(T^{\varepsilon} ; u, \varphi\right) & =2 \int_{\Omega^{\varepsilon}} m u\left(T^{\varepsilon}\right) d_{i j}\left(u^{\varepsilon}\right) d_{i j}(\varphi) d x \\
\left(p^{\varepsilon}, \operatorname{div} \varphi\right) & =\int_{\Omega^{\varepsilon}} p^{\varepsilon} d i v \varphi d x \\
j(\varphi) & =\int_{\omega} k^{\varepsilon}|\varphi| d x^{\prime}+\sqrt{2} \alpha^{\varepsilon} \int_{\Omega^{\varepsilon}}|D(\varphi)| d x \\
b\left(T^{\varepsilon}, \varphi\right) & =\int_{\Omega^{\varepsilon}} G^{\varepsilon}\left(\nabla T^{\varepsilon}\right)(\nabla \varphi) d x \\
c\left(u ; T^{\varepsilon}, \varphi\right) & =2 \int_{\Omega^{\varepsilon}} \mu\left(T^{\varepsilon}\right) d_{i j}(u) d_{i j}(u) \varphi d x+2 \alpha^{\varepsilon} \int_{\Omega^{\varepsilon}}|D(u)| \varphi d x+\int_{\Omega^{\varepsilon}} r^{\varepsilon}\left(T^{\varepsilon}\right) \varphi d x
\end{aligned}
$$

Theorem 1.1. Assume that $f^{\varepsilon} \in L^{2}\left(\Omega^{\varepsilon}\right)^{3}$ and $k^{\varepsilon} \in L_{+}^{\infty}(\omega)$; then there exists a unique $u^{\varepsilon} \in K_{\text {div }}^{\varepsilon}$, $T^{\varepsilon} \in H_{\Gamma_{1} \cup \Gamma_{L}}^{1}\left(\Omega^{\varepsilon}\right)$ and $p^{\varepsilon} \in L_{0}^{2}\left(\Omega^{\varepsilon}\right)$ (to an additive constant) solution to problem (2.11)-(2.12).

Proof. • For the proof of the equality $(2.11)$, see $[8,10]$.

- Now, for the equality (2.12), multiplying the equality (2.2) by $\varphi \in H_{\Gamma_{1} \cup \Gamma_{L}}^{1}\left(\Omega^{\varepsilon}\right)$ and by using Green's formula, we find

$$
\sum_{i=1}^{3} \int_{\Omega^{\varepsilon}} \frac{\partial T^{\varepsilon}}{\partial x_{i}} \frac{\partial \varphi}{\partial x_{i}} d x=\sum_{i, j=1}^{3} \int_{\Omega^{\varepsilon}} 2 \mu^{\varepsilon}\left(T^{\varepsilon}\right) D^{2}\left(u^{\varepsilon}\right) \varphi d x+\sqrt{2} \alpha^{\varepsilon} \int_{\Omega^{\varepsilon}} \frac{D^{2}\left(u^{\varepsilon}\right)}{\left|D\left(u^{\varepsilon}\right)\right|} \varphi d x+\int_{\Omega^{\varepsilon}} r^{\varepsilon}\left(T^{\varepsilon}\right) \varphi d x
$$

and as $D^{2}\left(u^{\varepsilon}\right)=\left|D\left(u^{\varepsilon}\right)\right|^{2}$, we obtain (2.12).

\section{Change of the domain and some estimates}

We shall now focus our attention on the asymptotic analysis of problem (2.1)-(2.9). For this, we transform this problem into an equivalent one on a domain $\Omega$ independent of the parameter $\varepsilon$ via the rescaling $z=\frac{x_{3}}{\varepsilon}$. So, for $\left(x^{\prime}, x_{3}\right)$ in $\Omega^{\varepsilon}$, we have $\left(x^{\prime}, z\right)$ in

$$
\Omega=\left\{\left(x^{\prime}, z\right) \in \mathbb{R}^{3},\left(x^{\prime}, 0\right) \in \omega \text { and } 0<z<h\left(x^{\prime}\right)\right\},
$$

and we denote by $\Gamma=\bar{\omega} \cup \Gamma_{L} \cup \Gamma_{1}$ its boundary, then we define the following functions in $\Omega$

$$
\hat{u}_{i}^{\varepsilon}\left(x^{\prime}, z\right)=u_{i}^{\varepsilon}\left(x^{\prime}, x_{3}\right), \quad i=1,2, \quad \hat{u}_{3}^{\varepsilon}\left(x^{\prime}, z\right)=\varepsilon^{-1} u_{3}^{\varepsilon}\left(x^{\prime}, x_{3}\right) \text { and } \hat{p}^{\varepsilon}\left(x^{\prime}, z\right)=\varepsilon^{2} p^{\varepsilon}\left(x^{\prime}, x_{3}\right) .
$$

Let us assume the following dependence (with respect of $\varepsilon$ ) of the data:

$$
\begin{gathered}
\hat{f}\left(x^{\prime}, z\right)=\varepsilon^{2} f^{\varepsilon}\left(x^{\prime}, x_{3}\right), \hat{\mu}=\mu^{\varepsilon}, \hat{\alpha}=\varepsilon \alpha^{\varepsilon}, \hat{l}=\varepsilon l^{\varepsilon} \text { and } \hat{k}=\varepsilon k^{\varepsilon} . \\
\widehat{G}=G^{\varepsilon}, \hat{r}=\varepsilon^{2} r^{\varepsilon} \text { and } T^{\varepsilon}\left(x^{\prime}, x_{3}\right)=\hat{T}^{\varepsilon}\left(x^{\prime}, z\right) .
\end{gathered}
$$


Let

$$
\begin{aligned}
K & =\left\{\hat{\phi} \in H^{1}(\Omega)^{3}: \hat{\phi}=0 \text { on } \Gamma_{L}, \quad \hat{\phi} . n=0 \text { on } \omega \cup \Gamma_{1}\right\}, \\
K_{d i v} & =\{\hat{\phi} \in K: \operatorname{div}(\hat{\phi})=0\}, \\
H_{\Gamma_{1} \cup \Gamma_{L}}^{1}(\Omega) & =\left\{\hat{\phi} \in H^{1}(\Omega): \hat{\phi}=0 \text { on } \Gamma_{1} \cup \Gamma_{L}\right\}, \\
V_{z} & =\left\{v=\left(v_{1}, v_{2}\right) \in L^{2}(\Omega)^{2}: \frac{\partial v_{i}}{\partial z} \in L^{2}(\Omega) ; v=0 \text { on } \Gamma_{L}\right\} .
\end{aligned}
$$

The norm of $V_{z}$ is $\|v\|_{V_{z}}=\left(\sum_{i=1}^{2}\left(\left\|v_{i}\right\|_{0, \Omega}^{2}+\left\|\frac{\partial v_{i}}{\partial z}\right\|_{0, \Omega}^{2}\right)\right)^{1 / 2}$.

By injecting the new data and unknown factors in (2.11)-(2.12) and after multiplication by $\varepsilon$, we deduce

$$
\begin{gathered}
\sum_{i, j=1}^{2} \int_{\Omega}\left[\varepsilon^{2} \mu\left(\frac{\partial \hat{u}_{i}^{\varepsilon}}{\partial x_{j}}+\frac{\partial \hat{u}_{j}^{\varepsilon}}{\partial x_{i}}\right)-\hat{p}^{\varepsilon} \delta_{i j}\right] \frac{\partial\left(\hat{\phi}_{i}-\hat{u}_{i}^{\varepsilon}\right)}{\partial x_{j}} d x^{\prime} d z+\sum_{i=1}^{2} \int_{\Omega} \mu\left(\frac{\partial \hat{u}_{i}^{\varepsilon}}{\partial z}+\varepsilon^{2} \frac{\partial \hat{u}_{3}^{\varepsilon}}{\partial x_{i}}\right) \frac{\partial\left(\hat{\phi}_{i}-\hat{u}_{i}^{\varepsilon}\right)}{\partial z} d x^{\prime} d z+ \\
+\int_{\Omega}\left(2 \mu \varepsilon^{2} \frac{\partial \hat{u}_{3}^{\varepsilon}}{\partial z}-\hat{p}^{\varepsilon}\right) \frac{\partial\left(\hat{\phi}_{3}-\hat{u}_{3}^{\varepsilon}\right)}{\partial z} d x^{\prime} d z+\sum_{j=1}^{2} \int_{\Omega} \mu \varepsilon^{2}\left(\varepsilon^{2} \frac{\partial \hat{u}_{3}^{\varepsilon}}{\partial x_{j}}+\frac{\partial \hat{u}_{j}^{\varepsilon}}{\partial z}\right) \frac{\partial\left(\hat{\phi}_{3}-\hat{u}_{3}^{\varepsilon}\right)}{\partial x_{j}} d x^{\prime} d z+ \\
+\sum_{i=1}^{2} \hat{l} \int_{\omega} \hat{u}_{i}^{\varepsilon}\left(x^{\prime}, h\left(x^{\prime}\right)\right)\left(\hat{\phi}_{i}\left(x^{\prime}, h\left(x^{\prime}\right)\right)-\hat{u}_{i}^{\varepsilon}\left(x^{\prime}, h\left(x^{\prime}\right)\right)\right) \sqrt{1+\left|\nabla h^{\varepsilon}\left(x^{\prime}\right)\right|^{2}} d x^{\prime}+ \\
+\int_{\omega} \hat{l}^{2} \hat{u}_{3}^{\varepsilon}\left(x^{\prime}, h\left(x^{\prime}\right)\right)\left(\hat{\phi}_{3}\left(x^{\prime}, h\left(x^{\prime}\right)\right)-\hat{u}_{3}^{\varepsilon}\left(x^{\prime}, h\left(x^{\prime}\right)\right)\right) \sqrt{1+\left|\nabla h^{\varepsilon}\left(x^{\prime}\right)\right|^{2}} d x^{\prime}+ \\
\quad+\int_{\omega} \hat{k}\left(|\hat{\phi}-s|-\left|\hat{u}^{\varepsilon}-s\right|\right) d x^{\prime}+\sqrt{2} \hat{\alpha} \int_{\Omega^{\varepsilon}}\left(|\widetilde{D}(\hat{\phi})|-\left|\widetilde{D}\left(\hat{u}^{\varepsilon}\right)\right|\right) d x^{\prime} d z \geqslant \\
\int_{\Omega}^{2}\left(\hat{f}_{i}, \hat{\phi}_{i}-\hat{u}_{i}^{\varepsilon}\right) d x^{\prime} d z+\int_{\Omega^{\varepsilon}} \varepsilon\left(\hat{f}_{3}, \hat{\phi}_{3}-\hat{u}_{3}^{\varepsilon}\right) d x^{\prime} d z . \\
\left.\quad+\sqrt{2} \hat{\alpha} \int_{\Omega} \mid \hat{T}^{\varepsilon}\right)\left(\nabla \hat{T}^{\varepsilon}\right)(\nabla \hat{\phi}) d x^{\prime} d z=2 \int_{\Omega} \hat{\mu}\left(\hat{T}^{\varepsilon}\right)\left|D\left(\hat{u}^{\varepsilon}\right)\right|^{2} \hat{\phi} d x^{\prime} d z+\int_{\Omega} \hat{r}\left(\hat{T}^{\varepsilon}\right) \hat{\phi} d x^{\prime} d z, \quad \forall \hat{\phi} \in H_{\Gamma_{1} \cup \Gamma_{L}}^{1}(\Omega)
\end{gathered}
$$

where

$$
|\widetilde{D}(v)|=\left[\frac{1}{4} \sum_{i, j=1}^{2} \varepsilon^{2}\left(\frac{\partial v_{i}}{\partial x_{j}}+\frac{\partial v_{j}}{\partial x_{i}}\right)^{2}+\frac{1}{2} \sum_{i=1}^{2}\left(\frac{\partial v_{i}}{\partial z}+\varepsilon^{2} \frac{\partial v_{3}}{\partial x_{i}}\right)^{2}+\varepsilon^{2}\left(\frac{\partial v_{3}}{\partial z}\right)^{2}\right]^{\frac{1}{2}}
$$

\subsection{A priori estimates on the velocity and the pressure}

In this subsection, we will obtain a priori estimates for the velocity field $\hat{u}^{\varepsilon}$ and the pressure $\hat{p}^{\varepsilon}$ in the domain $\Omega$. These estimates will be useful in proving the convergence of $\hat{u}^{\varepsilon}$ toward the expected function. However, it will not be enough to pass to the limit, and better estimates will be obtained in the next subsection. 
Theorem 2.1. Let the assumptions of Theorems 2.1 and 2.3 hold; then there exists a constant $C$ independent of $\varepsilon$ such that

$$
\begin{aligned}
\varepsilon^{2} \sum_{i, j=1}^{2}\left\|\frac{\partial \hat{u}_{i}^{\varepsilon}}{\partial x_{j}}\right\|_{0, \Omega}^{2}+ & \sum_{i=1}^{2}\left\|\frac{\partial \hat{u}_{i}^{\varepsilon}}{\partial z}\right\|_{0, \Omega}^{2}+\varepsilon^{2}\left\|\frac{\partial \hat{u}_{3}^{\varepsilon}}{\partial z}\right\|_{0, \Omega}^{2}+\varepsilon^{4} \sum_{i=1}^{2}\left\|\frac{\partial \hat{u}_{3}^{\varepsilon}}{\partial x_{i}}\right\|_{0, \Omega}^{2} \leqslant C, \\
& \left\|\hat{u}_{i}^{\varepsilon}\right\|_{0, \Omega} \leqslant C, \text { for } i=1,2, \\
& \left\|\varepsilon \hat{u}_{3}^{\varepsilon}\right\|_{0, \Omega} \leqslant C, \\
& \left\|\frac{\partial \hat{p}^{\varepsilon}}{\partial x_{i}}\right\|_{-1, \Omega} \leqslant C, \text { for } i=1,2, \\
& \left\|\frac{\partial \hat{p}^{\varepsilon}}{\partial z}\right\|_{-1, \Omega} \leqslant C \varepsilon .
\end{aligned}
$$

\subsection{A priori estimates on the temperature}

In this subsection, we look for a priori estimates on the temperature $\hat{T}^{\varepsilon}$, for this we need to establish the following Theorem:

Theorem 2.2. Under the same assumptions as in Theorem 3.1, there exists a constant $C$ independent of $\varepsilon$ such that

$$
\begin{gathered}
\sum_{i=1}^{2}\left\|\varepsilon \frac{\partial \hat{T}^{\varepsilon}}{\partial x_{i}}\right\|_{L^{2}(\Omega)}^{2} \leqslant C, \\
\left\|\frac{\partial \hat{T}^{\varepsilon}}{\partial z}\right\|_{L^{2}(\Omega)}^{2} \leqslant C .
\end{gathered}
$$

Proof. We choose in (3.2), $\hat{\phi}=\hat{T}^{\varepsilon}$ and by Korn inequality, we find

$$
\int_{\Omega} \varepsilon^{2} \widehat{G}\left(\hat{T}^{\varepsilon}\right) \nabla \hat{T}^{\varepsilon} \nabla \hat{T}^{\varepsilon} d x^{\prime} d z \geqslant \widehat{G} \varepsilon^{2}\left\|\nabla \hat{T}^{\varepsilon}\right\|_{L^{2}(\Omega)}^{2} \geqslant \widehat{G} \varepsilon^{2} \sum_{i=1}^{2}\left\|\frac{\partial \hat{T}^{\varepsilon}}{\partial x_{i}}\right\|_{L^{2}(\Omega)}^{2}+\widehat{G}\left\|\frac{\partial \hat{T}^{\varepsilon}}{\partial z}\right\|_{L^{2}(\Omega)}^{2}
$$

Let

$$
\begin{gathered}
I_{1}=2 \int_{\Omega} \hat{\mu}\left(\hat{T}^{\varepsilon}\right)\left|\widetilde{D}\left(\hat{u}^{\varepsilon}\right)\right|^{2} \hat{T}^{\varepsilon} d x^{\prime} d z \\
I_{2}=\sqrt{2} \hat{\alpha} \int_{\Omega}\left|\widetilde{D}\left(\hat{u}^{\varepsilon}\right)\right| \hat{T}^{\varepsilon} d x^{\prime} d z \text { and } I_{3}=\int_{\Omega} \hat{r}\left(\hat{T}^{\varepsilon}\right) \hat{T}^{\varepsilon} d x^{\prime} d z .
\end{gathered}
$$

By the Cauchy-Schwartz, Young inequalities and the compact injection $H^{1}(\Omega)$ in $L^{4}(\Omega)$, there exists a constant $C_{1}(\Omega)$ independent of $\varepsilon$, such that

$$
\left|I_{1}\right| \leqslant 2 \mu C_{4}(\Omega)\left[\begin{array}{c}
\sum_{i, j=1}^{2} \varepsilon^{2}\left\|\frac{\partial \hat{u}_{i}^{\varepsilon}}{\partial x_{j}}\right\|_{H^{1}(\Omega)}^{2}+\sum_{i=1}^{2}\left\|\frac{\partial \hat{u}_{i}^{\varepsilon}}{\partial z}\right\|_{H^{1}(\Omega)}^{2}+ \\
+\sum_{i=1}^{2} \varepsilon^{4}\left\|\frac{\partial \hat{u}_{3}^{\varepsilon}}{\partial x_{i}}\right\|_{H^{1}(\Omega)}^{2}+\varepsilon^{2}\left\|\frac{\partial \hat{u}_{3}^{\varepsilon}}{\partial z}\right\|_{H^{1}(\Omega)}^{2}
\end{array}\right]\left\|\hat{T}^{\varepsilon}\right\|_{L^{2}(\Omega)}
$$

So, using (3.3), we find: $\left|I_{1}\right| \leqslant 2 \mu C_{4}(\Omega) C\left\|\hat{T}^{\varepsilon}\right\|_{L^{2}(\Omega)}$.

Similarly,

$$
\left|I_{2}\right| \leqslant \sqrt{2} \hat{\alpha} C\left\|\hat{T}^{\varepsilon}\right\|_{L^{2}(\Omega)} \text { and }\left|I_{3}\right| \leqslant \widehat{r}_{\max } \bar{h}\left\|\frac{\partial \hat{T}^{\varepsilon}}{\partial z}\right\|_{L^{2}(\Omega)} \text {. }
$$


By injecting (3.11) in (3.10), it becomes

$$
\widehat{G} \varepsilon^{2} \sum_{i=1}^{2}\left\|\frac{\partial \hat{T}^{\varepsilon}}{\partial x_{i}}\right\|_{L^{2}(\Omega)}^{2}+\widehat{G}\left\|\frac{\partial \hat{T}^{\varepsilon}}{\partial z}\right\|_{L^{2}(\Omega)}^{2} \leqslant\left(2 \mu C_{4}(\Omega) C+\sqrt{2} \hat{\alpha} C+\widehat{r}_{\max } \bar{h}\right)\left\|\hat{T}^{\varepsilon}\right\|_{L^{2}(\Omega)} .
$$

As $\left\|\hat{T}^{\varepsilon}\right\|_{L^{2}(\Omega)} \leqslant \bar{h}\left\|\frac{\partial \hat{T}^{\varepsilon}}{\partial z}\right\|_{L^{2}(\Omega)}$, we find:

$$
\widehat{G} \varepsilon^{2} \sum_{i=1}^{2}\left\|\frac{\partial \hat{T}^{\varepsilon}}{\partial x_{i}}\right\|_{L^{2}(\Omega)}^{2}+\widehat{G}\left\|\frac{\partial \hat{T}^{\varepsilon}}{\partial z}\right\|_{L^{2}(\Omega)}^{2} \leqslant C_{5}\left\|\frac{\partial \hat{T}^{\varepsilon}}{\partial z}\right\|_{L^{2}(\Omega)}
$$

where: $C_{5}=\left(2 \mu C_{4}(\Omega) C+\sqrt{2} \hat{\alpha} C+\widehat{r}_{\max } \bar{h}\right) \bar{h}$.

According to (3.12) we deduce that : $\left\|\frac{\partial \hat{T}^{\varepsilon}}{\partial z}\right\|_{L^{2}(\Omega)} \leqslant C_{5} \widehat{G}^{-1}$.

By injecting this last estimate in (3.12), we deduce (3.8) and (3.9).

Theorem 2.3. Under the same assumptions as in Theorem 3.1, there exist $u^{\star}=\left(u_{1}^{\star}, u_{2}^{\star}\right) \in V_{z}$, $T^{*} \in V_{z}$ and $p^{\star} \in L_{0}^{2}(\Omega)$ such that:

$$
\begin{gathered}
\hat{u}_{i} \rightarrow u_{i}^{\star}, i=1,2 \text { weakly in } V_{z}, \\
\varepsilon \frac{\partial \hat{u}_{i}^{\varepsilon}}{\partial x_{j}} \rightarrow 0, i, j=1,2 \text { weakly in } L^{2}(\Omega), \\
\varepsilon \frac{\partial \hat{u}_{3}^{\varepsilon}}{\partial z} \rightarrow 0, \quad \text { weakly in } L^{2}(\Omega), \\
\varepsilon^{2} \frac{\partial \hat{u}_{3}^{\varepsilon}}{\partial x_{i}} \rightarrow 0, i=1,2 \text { weakly in } L^{2}(\Omega), \\
\hat{p}^{\varepsilon} \rightarrow p^{\star}, \text { weakly in } L^{2}(\Omega), p^{\star} \text { depend only of } x^{\prime} . \\
\varepsilon \hat{u}_{3}^{\varepsilon} \rightarrow 0, \quad \text { weakly in } L^{2}(\Omega), \\
\hat{T}^{\varepsilon} \rightarrow T^{*} \text { weakly in } V_{z} \\
\varepsilon \frac{\partial \hat{T}^{\varepsilon}}{\partial x_{i}} \rightarrow 0 \quad \text { weakly in } L^{2}(\Omega) .
\end{gathered}
$$

Proof. From (3.3) and (3.4), we deduce (3.13). Also (3.14) follow from (3.3) and (3.13). As $\operatorname{div}\left(\hat{u}^{\varepsilon}\right)=0$, by (3.14), we obtain (3.15). From (3.5) and (3.3), (3.16) hold. Using (3.6) and (3.7) we get (3.17). Because $\operatorname{div}\left(\hat{u}^{\varepsilon}\right)=0$, by (3.5) and with a particular choice of test function, we get (3.18). Finally, the convergences (3.19) and (3.20) are deduced directly from estimates $(3.8)-(3.9)$.

\section{Study of the limit problem}

In this section, using the second equation of (2.4) on $\Gamma_{1}^{\varepsilon}$, passing all non linear terms on the right and the linear terms on the left in the variational inequalities (3.1) and (3.2). Then, we apply the $\lim _{\varepsilon \rightarrow 0} \inf$ on the left and the $\lim _{\varepsilon \longrightarrow 0}$ on the right, using the convergence results of the Theorem 3.3, we deduce 


$$
\begin{aligned}
& \sum_{i=1}^{2} \int_{\Omega} \hat{\mu}\left(T^{*}\right) \frac{\partial u_{i}^{\star}}{\partial z} \frac{\partial\left(\hat{\phi}_{i}-u_{i}^{\star}\right)}{\partial z} d x^{\prime} d z-\int_{\omega} p^{\star}\left(x^{\prime}\right)\left(\sum_{i=1}^{2} \hat{\phi}_{i}\left(x^{\prime}, h\left(x^{\prime}\right)\right) \frac{\partial h}{\partial x_{i}}\right) d x^{\prime}- \\
& -\int_{\Omega} p^{\star}\left(x^{\prime}\right)\left(\frac{\partial \hat{\phi}_{1}}{\partial x_{1}}+\frac{\partial \hat{\phi}_{2}}{\partial x_{2}}\right) d x^{\prime} d z+\hat{l} \sum_{i=1}^{2} \int_{\omega} u_{i}^{\star}\left(x^{\prime}, h\left(x^{\prime}\right)\right)\left[\left(\hat{\phi}_{i}-u_{i}^{\star}\right)\left(x^{\prime}, h\left(x^{\prime}\right)\right)\right] d x^{\prime}+ \\
& +\hat{\alpha} \int_{\Omega}\left(\left|\frac{\partial \hat{\phi}}{\partial z}\right|-\left|\frac{\partial u^{\star}}{\partial z}\right|\right) d x^{\prime} d z+\hat{k} \int_{\omega}\left(|\hat{\phi}|-\left|u^{\star}\right|\right) d x^{\prime} \geqslant \sum_{j=1}^{2}\left(\hat{f}, \hat{\phi}-u^{\star}\right), \quad \forall \hat{\phi} \in \Pi(K), \\
& -\frac{\partial}{\partial z}\left(\widehat{G} \frac{\partial T^{*}}{\partial z}\right)=\sum_{i=1}^{2} \hat{\mu}\left(T^{*}\right)\left(\frac{\partial u_{i}^{*}}{\partial z}\right)^{2}+\sqrt{2} \hat{\alpha}\left|\frac{\partial u^{*}}{\partial z}\right|+\hat{r}\left(T^{*}\right), \quad \text { in } L^{2}(\Omega) .
\end{aligned}
$$

Moreover if

$$
\left.\left.\int_{\Omega}\left(\hat{\phi}_{1}\left(x^{\prime}, z\right)\right) \frac{\partial \theta}{\partial x_{1}}\left(x^{\prime}\right)+\hat{\phi}_{2}\left(x^{\prime}, z\right)\right) \frac{\partial \theta}{\partial x_{2}}\left(x^{\prime}\right)\right) d x^{\prime} d z=0, \quad \forall \theta \in C_{0}^{1}(\omega),
$$

then

$$
\begin{array}{r}
\sum_{i=1}^{2} \int_{\Omega} \hat{\mu}\left(T^{*}\right) \frac{\partial u_{i}^{\star}}{\partial z} \frac{\partial\left(\hat{\phi}_{i}-u_{i}^{\star}\right)}{\partial z} d x^{\prime} d z+\hat{l} \sum_{i=1}^{2} \int_{\omega} u_{i}^{\star}\left(x^{\prime}, h\left(x^{\prime}\right)\right)\left[\hat { \phi } _ { i } \left(x^{\prime}, h\left(x^{\prime}\right)-u_{i}^{\star}\left(x^{\prime}, h\left(x^{\prime}\right)\right] d x^{\prime}+\right.\right. \\
+\hat{\alpha} \int_{\Omega}\left(\left|\frac{\partial \hat{\phi}}{\partial z}\right|-\left|\frac{\partial u^{\star}}{\partial z}\right|\right) d x^{\prime} d z+\int_{\omega} \hat{k}\left(|\hat{\phi}|-\left|u^{\star}\right|\right) d x^{\prime} \geqslant \sum_{j=1}^{2}\left(\hat{f}, \hat{\phi}-u^{\star}\right),
\end{array}
$$

where $\Pi(K)=\left\{\bar{\phi}=\left(\hat{\phi}_{1}, \hat{\phi}_{2}\right) \in H^{1}(\Omega)^{2}: \exists \hat{\phi}_{3}\right.$ such that $\left.\phi=\left(\hat{\phi}_{1}, \hat{\phi}_{2}, \hat{\phi}_{3}\right) \in K\right\}$.

Theorem 3.1. The variational inequality (4.4) is equivalent the following system

$$
\begin{aligned}
\int_{\Omega} \hat{\mu}\left(T^{*}\right)\left|\frac{\partial u^{\star}}{\partial z}\right|^{2} d x^{\prime} d z+\hat{l} \int_{\omega} \mid u^{\star}\left(x^{\prime},\left.h\left(x^{\prime}\right)\right|^{2} d x^{\prime}\right. & +\int_{\omega} \hat{k}\left|u^{\star}\right| d x^{\prime}+ \\
& +\hat{\alpha} \int_{\Omega}\left|\frac{\partial u^{\star}}{\partial z}\right| d x^{\prime} d z-\int_{\Omega} \hat{f} u^{\star} d x^{\prime} d z=0
\end{aligned}
$$

and

$$
\begin{array}{r}
\int_{\Omega} \hat{\mu}\left(T^{*}\right) \frac{\partial u^{\star}}{\partial z} \frac{\partial \hat{\phi}}{\partial z} d x^{\prime} d z+\hat{l} \int_{\omega}\left(u^{\star} \hat{\phi}\right)\left(x^{\prime}, h\left(x^{\prime}\right) d x^{\prime}+\int_{\omega} \hat{k}|\hat{\phi}| d x^{\prime}+\hat{\alpha} \int_{\Omega}\left|\frac{\partial \hat{\phi}}{\partial z}\right| d x^{\prime} d z \geqslant\right. \\
\geqslant \int_{\Omega} \hat{f} \hat{\phi} d x^{\prime} d z, \forall \hat{\phi} \in \Sigma(K),
\end{array}
$$

where $\Sigma(K)=\{\bar{\phi} \in \Pi(K): \bar{\phi}$ satisfies condition (4.3) $\}$.

Proof. According to [3, Lemma 5.3] we can choose $\hat{\phi}=2 u^{\star}$ and $\hat{\phi}=0$ respectively in (4.4), to obtain (4.5).

For (4.6), we choose $\hat{\phi}=\left(\widehat{\psi}-u^{\star}\right)$ for all $\widehat{\psi} \in \Sigma(K)$.

Theorem 3.2. Let us set

$$
\sigma^{\star}=-\nabla p^{\star}+\widetilde{\sigma}^{\star} \text { and } \tilde{\sigma}^{\star}=\mu\left(T^{*}\right) \frac{\partial u^{\star}}{\partial z}+\hat{\alpha} \pi,
$$


$F\left(\hat{k} \hat{\phi}, \frac{\partial \hat{\phi}}{\partial z}\right)=\int_{\Omega} \mu\left(T^{*}\right) \frac{\partial u^{\star}}{\partial z} \frac{\partial \hat{\phi}}{\partial z} d x^{\prime} d z+\hat{l} \int_{\omega}\left(u^{\star} \hat{\phi}\right)\left(x^{\prime}, h\left(x^{\prime}\right) d x^{\prime}-\int_{\Omega} \hat{f} \hat{\phi} d x^{\prime} d z, \forall \hat{\phi} \in \Sigma(K)\right.$

then

$$
-\frac{\partial}{\partial z}\left[\mu\left(T^{*}\right) \frac{\partial u^{\star}}{\partial z}+\hat{\alpha} \frac{\partial u^{\star} / \partial z}{\left|\partial u^{\star} / \partial z\right|}\right]=\hat{f}-\nabla p^{\star} \text { in } L^{2}(\Omega)^{2},
$$

where $\pi$ obtained by the Hanh-Banach theorem, i.e. $\exists(\chi, \pi) \in L^{\infty}(\omega)^{2} \times L^{\infty}(\Omega)^{2}$, with $\|\chi\|_{\omega, \infty} \leqslant 1$, $\|\pi\|_{\Omega, \infty} \leqslant 1$, such that

$$
F\left(\hat{k} \hat{\psi}, \frac{\partial \hat{\psi}}{\partial z}\right)=-\int_{\omega} \chi \hat{k} \hat{\psi} d x^{\prime}-\hat{\alpha} \int_{\Omega} \pi \frac{\partial \hat{\psi}}{\partial z} d x^{\prime} d z
$$

Proof. If $\frac{\partial u^{\star}}{\partial z}=0$, from (4.7), we get $\left|\widetilde{\sigma}^{\star}\right| \leqslant \hat{\alpha}$.

In particular, from (4.5) and (4.8), we get

$$
\int_{\omega} \hat{k}\left|u^{\star}\right| d x^{\prime}+\hat{\alpha} \int_{\Omega}\left|\frac{\partial u^{\star}}{\partial z}\right| d x^{\prime} d z=\int_{\omega} \chi \hat{k} u^{\star} d x^{\prime}+\hat{\alpha} \int_{\Omega} \pi \frac{\partial u^{\star}}{\partial z} d x^{\prime} d z .
$$

Also, from (4.8) and (4.10), we have

$$
\begin{gathered}
\int_{\Omega} \mu\left(T^{*}\right) \frac{\partial u^{\star}}{\partial z} \frac{\partial \hat{\psi}}{\partial z} d x^{\prime} d z+\hat{l} \int_{\omega}\left(u^{\star} \hat{\psi}\right)\left(x^{\prime}, h\left(x^{\prime}\right) d x^{\prime}+\int_{\omega} \chi \hat{k} \hat{\psi} d x^{\prime}+\right. \\
+\hat{\alpha} \int_{\Omega} \pi \frac{\partial \hat{\psi}}{\partial z} d x^{\prime} d z-\int_{\Omega} \hat{f} \hat{\psi} d x^{\prime} d z=0, \forall \hat{\psi} \in \Sigma(K) .
\end{gathered}
$$

Now using (4.11), we have

$$
\hat{\alpha} \int_{\left|\frac{\partial u^{\star}}{\partial z}\right| \neq 0}\left(\left|\frac{\partial u^{\star}}{\partial z}\right|-\pi \frac{\partial u^{\star}}{\partial z}\right) d x^{\prime} d z+\int_{\omega} \hat{k}\left(\left|u^{\star}\right|-\chi u^{\star}\right) d x^{\prime}=0,
$$

since $\|\chi\|_{\omega, \infty} \leqslant 1$ and $\|\pi\|_{\Omega, \infty} \leqslant 1$, we deduce

$$
\left|\frac{\partial u^{\star}}{\partial z}\right|=\pi \frac{\partial u^{\star}}{\partial z} \text { and }\left|u^{\star}\right|=\chi u^{\star} .
$$

Hence, if $\left|\frac{\partial u^{\star}}{\partial z}\right| \neq 0$ by (4.7), we obtain

$$
\tilde{\sigma}^{\star}=\mu\left(T^{*}\right) \frac{\partial u^{\star}}{\partial z}+\hat{\alpha} \frac{\partial u^{\star} / \partial z}{\left|\partial u^{\star} / \partial z\right|} .
$$

In this case $\left|\widetilde{\sigma}^{\star}\right|=\left(\mu\left(T^{*}\right)+\frac{\hat{\alpha}}{\left|\partial u^{\star} / \partial z\right|}\right)\left|\frac{\partial u^{\star}}{\partial z}\right|=\mu\left(T^{*}\right)\left|\partial u^{\star} / \partial z\right|+\hat{\alpha}>\hat{\alpha}$.

Therefore, we can write

$$
\mu\left(T^{*}\right) \frac{\partial u^{\star}}{\partial z}=\left\{\begin{array}{l}
0 \quad \text { if }\left|\widetilde{\sigma}^{\star}\right| \leqslant \hat{\alpha} \\
\tilde{\sigma}^{\star}-\hat{\alpha} \frac{\partial u^{\star} / \partial z}{\left|\partial u^{\star} / \partial z\right|} \text { if }\left|\widetilde{\sigma}^{\star}\right|>\hat{\alpha}
\end{array}\right.
$$

which is a lower-dimensional Bingham law. 
Besides, from (4.12) there exists $p^{\star} \in L^{2}(\Omega)^{2}$ (see $\left.[5,13]\right)$ such that

$$
\begin{aligned}
\int_{\Omega} \mu\left(T^{*}\right) \frac{\partial u^{\star}}{\partial z} \frac{\partial \hat{\psi}}{\partial z} d x^{\prime} d z & +\hat{l} \int_{\omega}\left(u^{\star} \hat{\psi}\right)\left(x^{\prime}, h\left(x^{\prime}\right) d x^{\prime}+\int_{\omega} \underline{n} \hat{k} \hat{\psi} d x^{\prime}+\right. \\
& +\hat{\alpha} \int_{\Omega} \underline{m} \frac{\partial \hat{\psi}}{\partial z} d x^{\prime} d z-\int_{\Omega} \hat{f} \hat{\psi} d x^{\prime} d z=-\int_{\Omega} \nabla p^{\star} \hat{\psi} d x^{\prime} d z, \quad \forall \hat{\psi} \in \Pi(K) .
\end{aligned}
$$

Using (4.15)-(4.16) becomes

$$
\begin{aligned}
& \int_{\Omega} \mu\left(T^{*}\right) \tilde{\sigma}^{\star} \frac{\partial \hat{\psi}}{\partial z} d x^{\prime} d z+\hat{l} \int_{\omega}\left(u^{\star} \hat{\psi}\right)\left(x^{\prime},\right. h\left(x^{\prime}\right) d x^{\prime}+\int_{\omega} \underline{n} \hat{k} \hat{\psi} d x^{\prime}= \\
&=\int_{\Omega} \hat{f} \hat{\psi} d x^{\prime} d z-\int_{\Omega} \nabla p^{\star} \hat{\psi} d x^{\prime} d z, \forall \hat{\psi} \in \Pi(K),
\end{aligned}
$$

from which (4.9) follows if we take in (4.17) $\hat{\psi} \in H_{0}^{1}(\Omega)^{2}$.

Theorem 3.3. Under the assumptions of preceding theorems, the traces $s^{\star}, \tau^{\star}$ satisfy the following inequality

$$
\begin{aligned}
& \int_{\omega}\left(\frac{h^{3}}{12} \nabla p^{*}+\tilde{F}\left(x^{\prime}\right)+\right. \int_{0}^{h} \int_{0}^{y} \mu\left(T^{*}\left(x^{\prime}, \xi\right)\right) \frac{\partial u^{*}\left(x^{\prime}, \xi\right)}{\partial \xi} \partial \xi d y+ \\
&\left.+\hat{\alpha} \int_{0}^{h} \int_{0}^{y} \frac{\partial u^{*} / \partial \xi}{\left|\partial u^{*} / \partial \xi\right|}\left(x^{\prime}, \xi\right) d \xi d y\right) \nabla \varphi\left(x^{\prime}\right) d x^{\prime}-\int_{\omega} \frac{h}{2}\left(\int_{0}^{h} \mu\left(T^{*}\left(x^{\prime}, \xi\right)\right) \frac{\partial u^{*}\left(x^{\prime}, \xi\right)}{\partial \xi} \partial \xi-\right. \\
&\left.\quad-\frac{\hat{\alpha} h}{2} \int_{0}^{h} \frac{\partial u^{*} / \partial \xi}{\left|\partial u^{*} / \partial \xi\right|}\left(x^{\prime}, \xi\right) d \xi\right) \nabla \varphi\left(x^{\prime}\right) d x^{\prime}=0, \quad \forall \varphi \in H^{1}(\omega),
\end{aligned}
$$

where $\widetilde{F}\left(x^{\prime}\right)=\int_{0}^{h} F\left(x^{\prime}, y\right) d y-\frac{h}{2} F\left(x^{\prime}, h\right), \quad F\left(x^{\prime}, y\right)=\int_{0}^{y} \int_{0}^{\xi} \widehat{f}^{\varepsilon}\left(x^{\prime}, t\right) d t d \xi$.

Proof. We integrate twice (4.9) between 0 and $z$, to obtain

$$
\begin{aligned}
-\hat{\mu}\left(T^{*}\left(x^{\prime} ; z\right)\right) \frac{\partial u^{*}}{\partial z}\left(x^{\prime} ; z\right)-\hat{\alpha} \frac{\partial u^{*} / \partial z}{\left|\partial u^{*} / \partial z\right|}+\hat{\mu}\left(\zeta^{*}\left(x^{\prime}\right)\right) \tau^{*}\left(x^{\prime}\right)+\hat{\alpha} \frac{\tau^{*}}{\left|\tau^{*}\right|}= \\
=\int_{0}^{z} \hat{f}\left(x^{\prime}, \xi\right) d \xi-z \nabla p^{*}
\end{aligned}
$$

where, $\tau^{*}\left(x^{\prime}\right)=\frac{\partial u^{*}}{\partial z}\left(x^{\prime}, 0\right)$ and $\zeta^{*}\left(x^{\prime}\right)=T^{*}\left(x^{\prime}, 0\right)$.

By integrating between 0 in $z$, we find:

$$
\begin{aligned}
& -\int_{0}^{z} \hat{\mu}\left(T^{*}\left(x^{\prime} ; \xi\right)\right) \frac{\partial u^{*}}{\partial \xi}\left(x^{\prime} ; \xi\right) d \xi-\hat{\alpha} \int_{0}^{z} \frac{\partial u^{*} / \partial \xi}{\left|\partial u^{*} / \partial \xi\right|} d \xi+ \\
& \quad+\hat{\mu}\left(\zeta^{*}\left(x^{\prime}\right)\right) \tau^{*}\left(x^{\prime}\right) z+\hat{\alpha} \frac{\tau^{*}}{\left|\tau^{*}\right|} z=\int_{0}^{z} \int_{0}^{\xi} \hat{f}\left(x^{\prime}, y\right) d y d \xi-\frac{z^{2}}{2} \nabla p^{*}
\end{aligned}
$$

in particular for $z=h$ we obtain,

$$
\begin{aligned}
\left(\hat{\mu}\left(\zeta^{*}\left(x^{\prime}\right)\right) \tau^{*}\left(x^{\prime}\right) h+\hat{\alpha} \frac{\tau^{*}}{\left|\tau^{*}\right|} h\right) & \frac{h}{2}=\frac{h}{2} \int_{0}^{h} \hat{\mu}\left(T^{*}\left(x^{\prime} ; \xi\right)\right) \frac{\partial u^{*}}{\partial \xi}\left(x^{\prime} ; \xi\right) \xi+ \\
& +\hat{\alpha} \frac{h}{2} \int_{0}^{h} \frac{\partial u^{*} / \partial \xi}{\left|\partial u^{*} / \partial \xi\right|} d \xi+\frac{h}{2} \int_{0}^{h} \int_{0}^{\xi} \hat{f}\left(x^{\prime}, y\right) d y d \xi-\frac{h^{3}}{4} \nabla p^{*}
\end{aligned}
$$


integrating (4.20) between 0 and $h$, we obtain:

$$
\begin{aligned}
\left(\hat{\mu}\left(\zeta^{*}\left(x^{\prime}\right)\right) \tau^{*}\left(x^{\prime}\right) h+\right. & \left.\hat{\alpha} \frac{\tau^{*}}{\left|\tau^{*}\right|} h\right) \frac{h}{2}=\int_{0}^{h} \int_{0}^{y} \hat{\mu}\left(T^{*}\left(x^{\prime} ; \xi\right)\right) \frac{\partial u^{*}}{\partial z}\left(x^{\prime} ; \xi\right) d \xi d y+ \\
& +\hat{\alpha} \int_{0}^{h} \int_{0}^{y} \frac{\partial u^{*} / \partial \xi}{\left|\partial u^{*} / \partial \xi\right|} d \xi d y+\int_{0}^{h} \int_{0}^{y} \int_{0}^{\xi} \hat{f}\left(x^{\prime}, t\right) d t d \xi d y-\frac{h^{3}}{6} \nabla p^{*} .
\end{aligned}
$$

Substituting (4.21) into (4.22) and for all $\varphi \in H^{1}(\omega)$ we deduce (4.18).

For the uniqueness of the limit velocity and temperature, we put:

$$
\begin{aligned}
W_{z} & =\left\{u \in V_{z}: \frac{\partial^{2} u}{\partial z^{2}} \in L^{2}(\Omega)\right\}, \\
B_{c} & =\left\{u \in W_{z} \times W_{z}:\left\|\frac{\partial u}{\partial z}\right\|_{V_{z}} \leqslant c\right\}, \\
\tilde{W}_{z} & =\left\{u \in W_{z} \times W_{z}: u \text { satisfies condition (4.3) }\right\} .
\end{aligned}
$$

Theorem 3.4. The solution $\left(u^{*}, T^{*}\right)$ of the limit problem (4.2) and (4.5)-(4.6) is unique in $\left(\tilde{W}_{z} \cap B_{c}\right) \times W_{z}$, for all

$$
0<c<c_{0}=\left(2 C_{\hat{\mu}} \beta^{4}\right)^{-\frac{1}{2}}\left[\underline{G}\left[1+(\bar{h})^{2}\right]^{-1}-C_{\hat{r}}\right]^{\frac{1}{2}},
$$

where $\beta>0, C_{\hat{\mu}}>0, C_{\hat{r}}>0$ and $\underline{G}$ are determined in the proof.

Proof. For the proof of this theorem, we follow the same steps as in [2]. Let $\left(u^{\star, 1}, T^{*, 1}\right)$, $\left(u^{\star, 2}, T^{*, 2}\right)$ be two solutions of (4.2) and (4.5)-(4.6).

$$
\begin{aligned}
& \sum_{i=1}^{2} \int_{\Omega} \hat{\mu}\left(T^{1, *}\right) \frac{\partial u_{i}^{*, 1}}{\partial z} \frac{\partial\left(\hat{\varphi}_{i}-u_{i}^{*, 1}\right)}{\partial z} d x^{\prime} d z+ \\
& +\sum_{i=1}^{2} \hat{l} \int_{\omega} u_{i}^{*, 1}\left(x^{\prime}, h\left(x^{\prime}\right)\right)\left(\hat{\varphi}_{i}\left(x^{\prime}, h\left(x^{\prime}\right)\right)-u_{i}^{*, 1}\left(x^{\prime}, h\left(x^{\prime}\right)\right)\right) d x^{\prime}+\hat{\alpha} \int_{\Omega}\left(\left|D_{z}(\hat{\varphi})\right|-\left|D_{z}\left(u^{*, 1}\right)\right|\right) d x^{\prime} d z+ \\
& \quad+\int_{\omega} \hat{k}\left(|\hat{\varphi}-s|-\left|u^{*, 1}-s\right|\right) d x^{\prime} \geqslant \sum_{i=1}^{2} \int_{\Omega^{\varepsilon}}\left(\hat{f}_{i}, \hat{\varphi}_{i}-u_{i}^{*, 1}\right) d x^{\prime} d z \\
& +\sum_{i=1}^{2} \int_{\Omega} \hat{\mu}\left(T^{*, 2}\right) \frac{\partial u_{i}^{*, 2}}{\partial z} \frac{\partial\left(\hat{\varphi}_{i}-u_{i}^{*, 2}\right)}{\partial z} d x^{\prime} d z+ \\
& +\sum_{i=1}^{2} \hat{l} \int_{\omega} u_{i}^{*, 2}\left(x^{\prime}, h\left(x^{\prime}\right)\right)\left(\hat{\varphi}_{i}\left(x^{\prime}, h\left(x^{\prime}\right)\right)-u_{i}^{*, 2}\left(x^{\prime}, h\left(x^{\prime}\right)\right)\right) d x^{\prime}+\hat{\alpha} \int_{\Omega}\left(D_{z}(\hat{\varphi})-D_{z}\left(u^{*, 2}\right)\right) d x^{\prime} d z+ \\
& \quad+\int_{\omega} \hat{k}\left(|\hat{\varphi}-s|-\left|u^{*, 2}-s\right|\right) d x^{\prime} \geqslant \sum_{i=1}^{2} \int_{\Omega^{\varepsilon}}\left(\hat{f}_{i}, \hat{\varphi}_{i}-u_{i}^{*, 2}\right) d x^{\prime} d z
\end{aligned}
$$

where $D_{z}(\hat{\varphi})=\left(\sum_{i=1}^{2}\left(\frac{\partial \hat{\varphi}_{i}}{\partial z}\right)^{2}\right)^{\frac{1}{2}}$. Let us put $\hat{\varphi}=u^{*, 2}$ in (4.23) and $\hat{\varphi}=u^{*, 1}$ in (4.24), then adding two new equations, we find

$\sum_{i=1}^{2} \int_{\Omega}\left(\hat{\mu}\left(T^{*, 1}\right) \frac{\partial u_{i}^{*, 1}}{\partial z} \frac{\partial\left(u_{i}^{*, 2}-u_{i}^{*, 1}\right)}{\partial z}+\hat{\mu}\left(T^{*, 2}\right) \frac{\partial u_{i}^{*, 2}}{\partial z} \frac{\partial\left(u_{i}^{*, 1}-u_{i}^{*, 2}\right)}{\partial z}\right) d x^{\prime} d z \geqslant \hat{l} \sum_{i=1}^{2}\left\|u_{i}^{1}-u_{i}^{2}\right\|_{L^{2}(\omega)}^{2}$. 
As

$$
\begin{aligned}
& \sum_{i=1}^{2} \int_{\Omega}\left(\hat{\mu}\left(T^{*, 1}\right) \frac{\partial u_{i}^{*, 1}}{\partial z} \frac{\partial\left(u_{i}^{*, 2}-u_{i}^{*, 1}\right)}{\partial z}+\hat{\mu}\left(T^{*, 2}\right) \frac{\partial u_{i}^{*, 2}}{\partial z} \frac{\partial\left(u_{i}^{*, 1}-u_{i}^{*, 2}\right)}{\partial z}\right) d x^{\prime} d z= \\
= & -\sum_{i=1}^{2} \int_{\Omega} \hat{\mu}\left(T^{*, 1}\right)\left|\frac{\partial}{\partial z}\left(u_{i}^{*, 2}-u_{i}^{*, 1}\right)\right|^{2} d x^{\prime} d z+\sum_{i=1}^{2} \int_{\Omega}\left(\hat{\mu}\left(T^{*, 1}\right)-\hat{\mu}\left(T^{*, 2}\right)\right) \frac{\partial u_{i}^{*, 2}}{\partial z} \frac{\partial\left(u_{i}^{*, 1}-u_{i}^{*, 2}\right)}{\partial z} d x^{\prime} d z .
\end{aligned}
$$

then

$$
\begin{aligned}
\sum_{i=1}^{2} \int_{\Omega} \hat{\mu}\left(T^{*, 1}\right)\left|\frac{\partial}{\partial z}\left(u_{i}^{*, 2}-u_{i}^{*, 1}\right)\right|^{2} d x^{\prime} d z \leqslant & \leqslant \sum_{i=1}^{2} \int_{\Omega}\left[\hat{\mu}\left(T^{*, 1}\right)-\hat{\mu}\left(T^{*, 2}\right)\right] \frac{\partial u_{i}^{*, 2}}{\partial z} \frac{\partial\left(u_{i}^{*, 1}-u_{i}^{*, 2}\right)}{\partial z} d x^{\prime} d z .
\end{aligned}
$$

As $\hat{\mu} \geqslant \underline{\hat{\mu}}>0$ and using Poincaré's inequality, we have

$$
\sum_{i=1}^{2} \int_{\Omega} \hat{\mu}\left(T^{*, 1}\right)\left|\frac{\partial}{\partial z}\left(u_{i}^{*, 2}-u_{i}^{*, 1}\right)\right|^{2} d x^{\prime} d z \geqslant \mu_{*}\left[1+(\bar{h})^{2}\right]^{-1}\left\|u^{*, 2}-u^{*, 1}\right\|_{V_{z}}^{2} .
$$

Now, the analogous results of [2], is given by

$$
\left|\sum_{i=1}^{2} \int_{\Omega}\left(\hat{\mu}\left(T^{*, 1}\right)-\hat{\mu}\left(T^{*, 2}\right)\right) \frac{\partial u_{i}^{*, 2}}{\partial z} \frac{\partial\left(u_{i}^{*, 1}-u_{i}^{*, 2}\right)}{\partial z} d x^{\prime} d z\right| \leqslant \sqrt{2} \beta^{2} C_{\hat{\mu}} c\left\|T^{*, 1}-T^{*, 2}\right\|_{V_{z}}\left\|u^{*, 2}-u^{*, 1}\right\|_{V_{z}},
$$

where, $\beta>0, C_{\hat{\mu}}>0$ and $c>0$ are respectively deduced from, the embedding of $V_{z}$ in $L^{4}(\Omega)$, the assumption $\hat{\mu}$ is $C_{\hat{\mu}}$-Lipschitz continuous function on $\mathbb{R}$, and $u^{*, i} \in B_{c}$. Therefore

$$
\left\|u^{*, 2}-u^{*, 1}\right\|_{V_{z} \times V_{z}} \leqslant \sqrt{2} \beta^{2} C_{\hat{\mu}} \mu_{*}^{-1}\left[1+(\bar{h})^{2}\right] c\left\|T^{*, 1}-T^{*, 2}\right\|_{V_{z}} .
$$

On the other hand,

$$
\begin{aligned}
& \int_{\Omega} \widehat{G} \frac{\partial T^{*, 1}}{\partial z} \frac{\partial \hat{\psi}}{\partial z} d x^{\prime} d z= \\
& =\sum_{i=1}^{2} \int_{\Omega} \hat{\mu}\left(T^{*, 1}\right)\left(\frac{\partial u_{i}^{*, 1}}{\partial z}\right)^{2} \hat{\psi} d x^{\prime} d z+\sqrt{2} \hat{\alpha} \int_{\Omega}\left|D_{z}\left(u^{*, 1}\right)\right| \hat{\psi} d x^{\prime} d z+\int_{\Omega} \hat{r}\left(T^{*, 1}\right) \hat{\psi} d x^{\prime} d z \\
& \int_{\Omega} \widehat{G} \frac{\partial T^{*, 2}}{\partial z} \frac{\partial \hat{\psi}}{\partial z} d x^{\prime} d z= \\
& =\sum_{i=1}^{2} \int_{\Omega} \hat{\mu}\left(T^{*, 2}\right)\left(\frac{\partial u_{i}^{*, 2}}{\partial z}\right)^{2} \hat{\psi} d x^{\prime} d z+\sqrt{2} \hat{\alpha} \int_{\Omega}\left|D_{z}\left(u^{*, 2}\right)\right| \hat{\psi} d x^{\prime} d z+\int_{\Omega} \hat{r}\left(T^{*, 2}\right) \hat{\psi} d x^{\prime} d z
\end{aligned}
$$

By subtraction and choosing $\psi=\left(T^{*, 1}-T^{*, 2}\right) \in H_{\Gamma_{L} \cup \Gamma_{1}}^{1}(\Omega)$, we find

$$
\int_{\Omega} \widehat{G}\left|\frac{\partial}{\partial z}\left(T^{*, 1}-T^{*, 2}\right)\right|^{2} d x^{\prime} d z=\sum_{k=1}^{4} I_{k}
$$


where

$$
\begin{aligned}
& I_{1}=\sum_{j=1}^{2} I_{1}^{j}, \quad I_{1}^{j}=\int_{\Omega} \hat{\mu}\left(T^{*, 1}\right) \frac{\partial}{\partial z}\left(u_{i}^{*, 1}+u_{i}^{*, 2}\right) \frac{\partial}{\partial z}\left(u_{i}^{*, 1}-u_{i}^{*, 2}\right)\left(T^{*, 1}-T^{*, 2}\right) d x^{\prime} d z \\
& I_{2}=\sum_{j=1}^{2} I_{2}^{j}, \quad I_{2}^{j}=\int_{\Omega}\left[\hat{\mu}\left(T^{*, 1}\right)-\hat{\mu}\left(T^{*, 2}\right)\right]\left(\frac{\partial u_{i}^{*, 2}}{\partial z}\right)^{2}\left(T^{*, 1}-T^{*, 2}\right) d x^{\prime} d z \\
& I_{3}=\int_{\Omega}\left(\hat{r}\left(T^{*, 1}\right)-\hat{r}\left(T^{*, 2}\right)\right)\left(T^{*, 1}-T^{*, 2}\right) d x^{\prime} d z \\
& I_{4}=\sqrt{2} \hat{\alpha} \int_{\Omega}\left(D_{z}\left(u^{*, 1}\right)-D_{z}\left(u^{*, 2}\right)\right)\left(T^{*, 1}-T^{*, 2}\right) d x^{\prime} d z .
\end{aligned}
$$

The increases of $I_{k}, k=1,2,3$ are given by [2] as follows

$$
\begin{aligned}
I_{1} & =\left|\sum_{j=1}^{2} I_{1}^{j}\right| \leqslant 2 \sqrt{2} \mu^{*} \beta^{2} c\left\|u_{i}^{*, 1}-u_{i}^{*, 2}\right\|_{V_{z} \times V_{z}}\left\|T^{*, 1}-T^{*, 2}\right\|_{V_{z}}, \\
\left|I_{2}^{i}\right| & \leqslant C_{\hat{\mu}} \beta^{4}\left\|T^{*, 1}-T^{*, 2}\right\|_{V_{z}}^{2}\left\|u_{i}^{2}\right\|_{W_{z}}^{2}, \\
\left|I_{3}\right| & \leqslant C_{\hat{r}}\left\|T^{*, 1}-T^{*, 2}\right\|_{V_{z}}^{2},
\end{aligned}
$$

where $C_{\hat{r}}>0$ deducted from the assumption $\hat{r}$ is $C_{\hat{r}}$-Lipschitz continuous function on $\mathbb{R}$. Using the Cauchy-Schwartz inequality, we obtain:

$$
\left|I_{4}\right| \leqslant 2 \hat{\alpha}\left\|T^{*, 1}-T^{*, 2}\right\|_{V_{z}}^{2}\left\|u^{*, 2}-u^{*, 1}\right\|_{V_{z} \times V_{z}} .
$$

Injecting (4.31)-(4.34) in (4.30), we find:

$$
\begin{aligned}
& \underline{G}\left[1+(\bar{h})^{2}\right]^{-1}\left\|T^{*, 1}-T^{*, 2}\right\|_{V_{z}}^{2} \leqslant 2 \sqrt{2} \mu^{*} \beta^{2} c\left\|u_{i}^{*, 1}-u_{i}^{*, 2}\right\|_{V_{z} \times V_{z}}\left\|T^{*, 1}-T^{*, 2}\right\|_{V_{z}}+ \\
& \quad+C_{\hat{\mu}} \beta^{4} c^{2}\left\|T^{*, 1}-T^{*, 2}\right\|_{V_{z}}^{2}+C_{\hat{r}}\left\|T^{*, 1}-T^{*, 2}\right\|_{V_{z}}^{2}+2 \hat{\alpha}\left\|T^{*, 1}-T^{*, 2}\right\|_{V_{z}}^{2}\left\|u^{*, 2}-u^{*, 1}\right\|_{V_{z} \times V_{z}}
\end{aligned}
$$

SO

$$
\left\|T^{*, 1}-T^{*, 2}\right\|_{V_{z}} \leqslant\left[\underline{G}\left[1+(\bar{h})^{2}\right]^{-1}-2 C_{\hat{\mu}} \beta^{4} c^{2}-C_{\hat{r}}\right]^{-1}\left[2 \sqrt{2} \bar{\mu} \beta^{2} c+2 \hat{\alpha}\right]\left\|u^{*, 2}-u^{*, 1}\right\|_{V_{z} \times V_{z}} .
$$

where, $\underline{\hat{\mu}}<\hat{\mu}<\overline{\hat{\mu}}$, and $\underline{G}=\min \widehat{G}$. It is assumed that:

$$
\begin{aligned}
& 0<c<c_{0}=\left(2 C_{\hat{\mu}} \beta^{4}\right)^{-\frac{1}{2}}\left[\underline{G}\left[1+(\bar{h})^{2}\right]^{-1}-C_{\hat{r}}\right]^{\frac{1}{2}}, \\
& \underline{G}>\left[1+(\bar{h})^{2}\right] C_{\hat{r}}
\end{aligned}
$$

Therefore,

$$
\left\|T^{*, 1}-T^{*, 2}\right\|_{V_{z}} \leqslant\left(2 \sqrt{2} \bar{\mu} \beta^{2} c+2 \hat{\alpha}\right)\left(c_{0}^{2}-c^{2}\right)^{-1}\left\|u^{*, 2}-u^{*, 1}\right\|_{V_{z} \times V_{z}} .
$$

Now, Injecting (4.27) in (4.35), we obtain:

$$
\left(1-\left(2 \sqrt{2} \bar{\mu} \beta^{2} c+2 \hat{\alpha}\right)\left(c_{0}^{2}-c^{2}\right)^{-1} \sqrt{2} \beta^{2} C_{\hat{\mu}}\left(\underline{\mu}^{-1}\right)\left[1+(\bar{h})^{2}\right] c\right)\left\|T^{*, 1}-T^{*, 2}\right\|_{V_{z}} \leqslant 0
$$


assuming that

$$
\left(1-\left(2 \sqrt{2} \bar{\mu} \beta^{2} c+2 \hat{\alpha}\right)\left(c_{0}^{2}-c^{2}\right)^{-1} \sqrt{2} \beta^{2} C_{\hat{\mu}}\left(\underline{\mu}^{-1}\right)\left[1+(\bar{h})^{2}\right] c\right)>0
$$

we have

$$
\left\|T^{*, 1}-T^{*, 2}\right\|_{V_{z}}=0
$$

According to (4.27), we deduce

$$
\left\|u^{*, 2}-u^{*, 1}\right\|_{V_{z} \times V_{z}}^{2} \leqslant 0 .
$$

Then $u^{*, 2}=u^{*, 1}$ almost everywhere in $V_{z} \times V_{z}$. This completes the proof.

\section{References}

[1] D.Benterki, H.Benseridi, M.Dilmi, Asymptotic Study of a Boundary Value Problem Governed by the Elasticity Operator with Nonlinear Term, Adv. Appl. Math. Mech., 6(2014), 191-202.

[2] M.Boukrouche, F.Saidi, Non-isothermal lubrication problem with Tresca fluid-solid interface law. Part II, Asymptotic behavior of weak solutions, Nonlinear Analysis: Real World Applications, 9(2008), no. 4, 1680-1701.

[3] M.Boukrouche, G.Łukaszewicz, On a lubrication problem with Fourier and Tresca boundary conditions, Math. Mod. and Meth. in Applied Sciences, 14(2004), no. 6, 913-941.

[4] M.Boukrouche, R. El mir, Asymptotic analysis of non-Newtonian fluid in a thin domain with Tresca law, Nonlinear analysis, Theory Methods and Applications. 59 (2004), 85-105.

[5] R.Bunoui, S.Kesavan, Asymptotic behaviour of a Bingham fluid in thin layers, J. Math. Anal. Appl., 293(2004), no. 2, 405-418.

[6] E.J.Dean, R.Glowinski, G.Guidoboni, On the numerical simulation of bingham visco-plastic flow: Old and new results, Journal of Non-Newtonian Fluid Mechanics, 142(2007), 36-62.

[7] J.C.De Los Reyes, S.Gonz alez, Path following methods for steady laminar Bingham flow in cylindrical pipes, M2AN Math. Model. Numer. Anal., 43(2009), no. 1, 81-117.

[8] M.Dilmi, H.Benseridi, A.Saadallah, Asymptotic Analysis of a Bingham Fluid in a Thin Domain with Fourier and Tresca Boundary Conditions, Adv. Appl. Math. Mech., 6(2014), $797-810$.

[9] G.Duvant, J.L.Lions, Les inéquations en mécanique et en physique, Dunod, Paris, 1972.

[10] R.Elmir, Comportement asymptotique d'un fluide de Bingham dans un filmi mince avec des conditions non-linéaires sur le bord, Thèse de Doctorat, Chapitre 4, Université Saint Etienne, France, 2006.

[11] M.Fuchs, J.F.Grotowski, J.Reuling, On variational model for quasi-static Bingham fluids, Math. Meth. and Meth. in Applied Sciences, 19(1996), 991-1015.

[12] M.Fuchs, G.Seregin, Regularity results for the quasi-static Bingham variational inequality in dimensions two and three, Mathematische Zeitschrift, 227(1998), 525-541.R.

[13] R.Glowinski, J.L.Lions, and R.Tremolieres, Analyse numerique des inequations variationnelles, Tome 1: Theorie generale premieres applications, Methodes Mathematiques de l'Informatique, Vol. 5, Dunod, Paris, 1976 


\title{
Исследование проблемы неизотермической связи со смешанными граничными условиями в тонком домене с законом трения
}

\author{
Абделкадер Саадалах \\ Хамид Бенсериди \\ Моурад Дилми \\ Лаборатория прикладной математики, Отдел математики \\ Университет Ферхата Аббаса Сетифа 1 \\ Сетиф, 19000 \\ Алжир
}

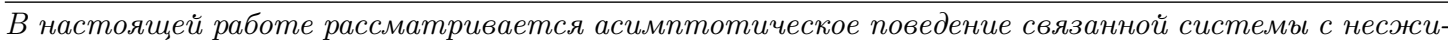
маемой жидкостъю Бингхэма и уравнения тепловой энергии в трехмерной ограниченной области с условиями свободного трения Треска. Во-первых, мы доказываеме результаты существования и единственности для слабого решения. Во-вторых, мы показываем сильную сходимость скорости и температуры. Затем получаем конкретное предельное уравнение Рейнольдса и доказываем единственность предельной скорости и температуры.

Ключевые слова: асимптотический подход, граничные условия, сопряженная задача, закон Фуръе, неизотермическая жидкость Бингама, закон Треска, уравнение Рейнольдса. 